\title{
Editorial
}

Psychotherapeut 2015 - 60:93

DOI 10.1007/s00278-015-0015-x

Online publiziert: 19. März 2015

(c) Springer-Verlag Berlin Heidelberg 2015

\author{
Manfred Cierpka ${ }^{1}$. Verena Kast ${ }^{2}$ - Peter Henningsen ${ }^{3}$ \\ ${ }^{1}$ Institut für Psychosomatische Kooperationsforschung und Familientherapie, \\ Psychosoziales Zentrum, Uniklinikum Heidelberg, Heidelberg, Deutschland \\ ${ }^{2}$ C.G.-Jung-Institut Zürich, Zürich, Schweiz \\ ${ }^{3}$ Klinik für Psychosomatische Medizin und Psychotherapie, Klinikum rechts der Isar, TU München, \\ München, Deutschland
}

\section{Zeiterfahrung und Schicksal}

Das zweite Leitthema der Tagung be-

Die Schwerpunkte dieses Hefts der Zeitschrift Psychotherapeut entsprechen den Leitthemen der Lindauer Psychotherapiewochen 2014.

"Zeit“ war das Leitthema der ersten Tagungswoche. Zeit ist nicht nur im Leben kostbar als Lebenszeit, sondern auch in der Psychotherapie als Behandlungszeit. Weil die Zeit begrenzt ist, überlegen Psychotherapeuten, wie sie sie am besten nutzen. Sie richten sich nach den Patienten, die entsprechend ihrem Störungsbild in der Behandlung mehr oder weniger Zeit für Veränderungen benötigen. Häufig nehmen Entwicklungen auch einen phasenweisen Verlauf; Zeiten der Veränderung folgen Ruhepausen und Stabilisierungsphasen.

Der Umgang mit der Zeit berührt grundlegende Fragen der Psychotherapie. Nicht nur die Dosis als Zahl der Stunden, auch die Frequenz und die Dauer sind maßgebend für persönliche Veränderung. Dorothea Huber diskutiert in ihrer Übersichtsarbeit die Ergebnisse aus der Psychotherapieforschung, die uns bei den Überlegungen helfen können, in welchem Fall eine Kurz- und in welchem Fall eine Langzeitpsychotherapie indiziert ist. Was wissen wir heute über Dosis-Wirkung-Modelle, über Unterschiede in den Ergebnissen beim Patienten nach einer Langzeitgegenüber einer Kurzzeittherapie?

Es folgen 3 Originalarbeiten zu diesem Schwerpunkt. Thomas Fuchs schreibt über das Zeiterleben in Gesundheit und Krankheit, und Verena Kast referiert aus klinischer Sicht über das Phänomen des Zeiterlebens im Traum. Diana Pflichthofer macht deutlich, dass das Lernen von und in der Psychotherapie ein langfristiger Bildungsprozess ist, den sie als „Wanderjahre" charakterisiert. schäftigte sich mit dem „Schicksal“, wie es uns im Leben und in der Psychotherapie begegnet. In unserem Leben ist nicht alles planbar; einiges muss auch einfach hingenommen werden, z. B. unser Körper, die Eltern, aber auch die Zeit, in die wir hineingeboren werden u.v.m. Wie gehen wir in der Psychotherapie, die doch so sehr auf Veränderung aus ist, mit den existenziellen Situationen um, die nicht leicht zu verändern sind, mit der Spannung zwischen Freiheit und Schicksal? Wie gelingt es, angesichts des Unabänderlichen sich doch eine gewisse Freiheit zu bewahren? Auch als Leidende bleibt uns die Freiheit, eine Einstellung zum Leiden zu finden.

Einige für die Publikation überarbeitete Kongressbeiträge werden in dieser Ausgabe von Psychotherapeut veröffentlicht. Giovanni Maio mahnt in seinem Beitrag eine Bereitschaft an, das einem Widerfahrene anzunehmen, sich im Vorgegebenen einzurichten und gerade dadurch fähig zu werden, das Leben aktiv zu gestalten. Das gelte auch für die Haltung in der Therapie. Eckhard Frick setzt sich mit dem Begriff des Schicksals aus mehreren Perspektiven auseinander und verortet ihn dort, wo wir an die Grenzen der Kontrollmöglichkeiten und der eigenen Kompetenz gelangen. Therapeutisch fordert er die Anerkennung der eigenen Verwundbarkeit im therapeutischen Handeln. Annette Streeck-Fischer fragt: Ist das Aufmerksamkeitsdefizit- und Hyperaktivitätssyndrom (ADHS) Schicksal? Schicksal, so meint sie, ist es, ob man Behandler findet, die die Komplexität dieser Störung wahrnehmen und entsprechend therapeutisch handeln können. Dieser grundlegende Beitrag wird helfen, die Komplexität dieser Störung zu sehen und zu verstehen.
Wir bedanken uns sehr bei den Vortragenden, die sich bereit erklärten, ihre Vorträge zu wissenschaftlichen Manuskripten zu überarbeiten. Dieses Heft erscheint rechtzeitig zu den Lindauer Psychotherapiewochen 2015 als Nachlese. Wir wünschen allen Lesern eine interessante Lektüre.

\section{Korrespondenzadresse}

\section{Prof. Dr. M. Cierpka}

Institut für Psychosomatische

Kooperationsforschung und Familientherapie

Psychosoziales Zentrum, Uniklinikum

Heidelberg, Heidelberg

manfred_cierpka@med.uni-heidelberg.de

\section{Einhaltung ethischer Richtlinien}

Interessenkonflikt. M. Cierpka, V. Kast und P. Henningsen geben an, dass kein Interessenkonflikt besteht.

Dieser Beitrag beinhaltet keine Studien an Menschen oder Tieren. 